\title{
EDITORIAL
}

\section{Evidence supports the superiority of closed ICUs for patients and families: we are not sure}

\author{
B. Guidet ${ }^{1,2,3^{*}}$, N. Kentish-Barnes ${ }^{4}$ and H. Wunsch ${ }^{5,6}$
}

๑ 2016 Springer-Verlag Berlin Heidelberg and ESICM

Intensive care units (ICUs) are unique locations in the hospital. Very few countries have set minimum criteria to qualify a unit as an ICU [1]. What is clear is that an ICU is not merely a place where specific procedures are performed. Patients can be mechanically ventilated in the post-anesthesia care unit; renal replacement therapy is a common practice in nephrology units; and vasopressors are routinely used in coronary care units. An intensive care unit is a specific entity with unique characteristics. It gathers people with different backgrounds (physicians, nurses, different therapists, clerks, etc.) working $24 \mathrm{~h}$ a day taking care of critically ill patients, and the care provided encompasses many aspects of medicine, including ethical concerns. As a consequence, working as a team with a shared culture of care is of paramount importance [2].

A major concern regarding the open model of organization is that it is a model that is counter to this idea of a team approach, with different professionals coming from different backgrounds and more likely to have different goals without a shared culture. Why are we still "on the fence" regarding the importance of closed ICUs? Several aspects warrant discussion.

1. Focusing on mortality for ICU patients is overly simplistic $[3,4]$.

Mortality rates can be influenced by numerous external factors and questions remain as to the best moment to assess mortality? Many factors besides

\footnotetext{
*Correspondence: bertrand.quidet@aphp.fr

1 Service de Réanimation Médicale, Hôpital Saint-Antoine, Assistance Publique-Hôpitaux de Paris (AP-HP), 75012 Paris, France

Full author information is available at the end of the article
}

For contrasting viewpoints, please go to doi:10.1007/s00134-016-4438-9 and doi:10.1007/s00134-016-4466-5. quality of the care delivered impact mortality, mainly related to case mix, admission and discharge policies and hospital organization. A recently raised concern regarding measurement of mortality is the impact of end-of-life care preferences, and how they may alter the interpretation of mortality as an outcome [5]. Other outcomes are also a consideration such as length of stay, time of day patients are discharged, and long-term functional status and quality of life. To adequately interpret the existing literature, we must take into account the many potential confounders of patient characteristics, and the organization of ICUs and hospitals.

2. Understanding culture and communication is not straightforward.

Traumatic, demanding and emotional events are frequent in the ICU. In this context, caregivers are at risk of developing stress and burnout symptoms [6]. However, they may better cope with these situations in the long term if they work in an environment that emphasizes trust and support [7], and in a climate where relationships between different caregivers is based on respect and knowledge of each other's personalities and competence. A well-functioning team with good leadership, open communication and a good atmosphere are key elements that will impact on work commitment [8], problem-solving, quality end-of-life decision-making and experience of all participants. In this regard, closed ICUs may make it easier to promote teambuilding than in an open ICU model where physicians may come and go more frequently. The need for coherent decisionmaking is vital for caregivers: being able to express 
oneself, to ask questions or to disagree is more likely when one feels secure and free to do so during a well-coordinated discussion. Having to discuss emotional decisions with colleagues or experts one barely knows can make the exercise difficult and can hinder the shared decision-making model.

Patients' relatives appreciate a caring atmosphere in which they receive continuous, straightforward and honest information [9]. In an open ICU, it may sometimes be difficult to designate a legitimate discussion coordinator. However, on the flip side, patients and families may find it easier to have discussions of difficult decisions in the ICU with physicians they have met prior to ICU admission; these physicians may know them and their care preferences better than an intensivist meeting them for the first time at the door of the ICU. Given the difficulty of end-of-life decision-making, finding ways to minimize this stress is important.

3. Information on non-mortality patient-centered outcomes is lacking.

With the focus on risk-adjusted mortality, few studies have fully examined the patient and family experience in closed versus open ICUs, and much of the impact of closed ICUs also remains speculative [10].

A recent scoping study found 224 articles related to the transition of patients out of intensive care [10]. Recognizing that this transition may be traumatic, particularly when leaving a "closed" ICU, some hospitals have instituted a follow-up or transition team to check on patients after ICU discharge [11]. In this regard, open ICUs may offer a continuity for patients and families that closed ICUs do not-a physician the patient and family know is still there taking care of them despite the change of scenery within the hospital; therefore, survivors of critical illness (and particularly their families) may view the experiences through a different lens from patients and families who experience end-of-life decision-making in the ICU.

There are two issues relevant to the care provided in a closed or open model. The first is that, when the ICU becomes a "black box" of care, the experience of the patient and their potential needs may be more easily lost in the shuffle of the transition out of the hospital [12]. To this end, many institutions have embraced the idea of an ICU follow-up clinic to specifically address these concerns. To date, the largest randomized controlled trial on the topic for patients cared for in closed ICU settings (Scotland) did not demonstrate improvements in outcomes [13]. We know very little about whether the post-hospital care for patients cared for in open ICUs allows for better quality of post-hospital care. The second issue is the ability to "feedback" what is now known about outcomes for our patients after critical illness to try to improve care delivered during the peak of critical illness [12, 14]. As mentioned above regarding the potential benefits of a team environment in the ICU, such potential change may be easier in a closed unit with individual physicians who are invested in the ICU experience rather than focused on care throughout the hospital.

No information is available on whether closed ICUs versus open ICUs perform better for: resident's training, knowledge transfer, role modeling, conflict resolution, and leadership.

The open model is widely used in the US while the closed model is common practice in Europe. It is important to note that most of the literature on this topic is from the US. The Society of Critical Care Medicine states that "an intensivist-led, high-intensity team is an integral part of effective care delivery in the ICU" and that "there does not appear to be any additional benefit from $24 / 7$ intensivist staffing within a high-intensity staffing model" [15]. It is hard to generalize conclusions originating from one country to other countries and particularly to middle or low-income countries. The pressures within the US, such as a relative shortage of board-certified ICU physicians and higher physician incomes compared with many European countries, may impact the staffing models chosen.

\footnotetext{
Author details

' Service de Réanimation Médicale, Hôpital Saint-Antoine, Assistance Publique-Hôpitaux de Paris (AP-HP), 75012 Paris, France. ${ }^{2}$ Sorbonne Universités, Université Pierre et Marie Curie -Paris 06, Paris, France. ${ }^{3}$ Institut National de la Santé et de la Recherche Médicale (INSERM), UMR_S 1136, Institut Pierre Louis d'Épidémiologie et de Santé Publique, 75013 Paris, France. ${ }^{4}$ Famiréa Research Group, Medical Intensive Care Unit, Saint-Louis University Hospital, Paris, France. ${ }^{5}$ Department of Critical Care Medicine, Sunnybrook Health Sciences Center, Toronto, ON, Canada. ${ }^{6}$ Department of Anesthesia and Interdepartmental Division of Critical Care Medicine, University of Toronto, Toronto, ON, Canada.
}

Received: 5 August 2016 Accepted: 15 August 2016 Published online: 1 September 2016

References

1. Valentin A, Ferdinande P, ESICM Working Group on Quality Improvement (2011) Recommendations on basic requirements for intensive care units: structural and organizational aspects. Intensive Care Med 37:1575-1587 
2. Guidet B, González-Romá V (2011) How climate and cultural aspects influence performance in the Intensive care units? Crit Care 15:312

3. Weissman GE, Halpern SD (2016) Evidence supports the superiority of closed ICUs for patients and families: no. Intensive Care Med. doi:10.1007/ s00134-016-4438-9

4. Vincent JL (2016) Evidence supports the superiority of closed ICUs for patients and families: yes. Intensive Care Med. doi:10.1007/ s00134-016-4466-5

5. Walkey AJ, Weinberg J, Wiener RS, Cooke CR, Lindenauer PK (2016) Association of do-not-resuscitate orders and hospital mortality rate among patients with pneumonia. JAMA Intern Med 176(1):97-104

6. Embriaco N, Azoulay E, Barrau K, Kentish N, Pochard F, Loundou A, Papazian L (2007) High level of burnout in intensivists: prevalence and associated factors. Am J Respir Crit Care Med 175:686-692

7. Wåhlin I, Ek AC, Idvall E (2010) Staff empowerment in intensive care: nurses' and physicians' lived experiences. Intensive Crit Care Nurs 26:262-269

8. Laschinger HK, Finegan J, Wilk P (2009) Context matters: the impact of unit leadership and empowerment on nurses' organizational commitment. J Nurs Adm 39:228-235

9. Azoulay E, Sprung CL (2004) Family-physician interactions in the intensive care unit. Crit Care Med 32:2323-2328

10. Stelfox HT, Lane D, Boyd JM, Taylor S, Perrier L, Straus S, Zygun D, Zuege DJ (2015) A scoping review of patient discharge from intensive care: opportunities and tools to improve care. Chest 147:317-327

11. Niven DJ, Bastos JF, Stelfox HT (2014) Critical care transition programs and the risk of readmission or death after discharge from an ICU: a systematic review and meta-analysis. Critical Care Med 42:179-187
12. Needham DM, Davidson J, Cohen H, Hopkins RO, Weinert C, Wunsch H, Zawistowski C, Bemis-Dougherty A, Berney SC, Bienvenu OJ, Brady SL, Brodsky MB, Denehy L, Elliott D, Flatley C, Harabin AL, Jones C, Louis D, Meltzer W, Muldoon SR, Palmer JB, Perme C, Robinson M, Schmidt DM, Scruth E, Spill GR, Storey CP, Render M, Votto J, Harvey MA (2012) Improving long-term outcomes after discharge from intensive care unit: report from a stakeholders' conference. Critical Care Med 40:502-509

13. Cuthbertson BH, Rattray J, Campbell MK, Gager M, Roughton S, Smith A, Hull A, Breeman S, Norrie J, Jenkinson D, Hernández R, Johnston M, Wilson E, Waldmann C, PRaCTICaL study group (2009) The PRaCTICaL study of nurse led, intensive care follow-up programmes for improving long term outcomes from critical illness: a pragmatic randomised controlled trial. BMJ 339:b3723

14. Herridge MS, Tansey CM, Matté A, Tomlinson G, Diaz-Granados N, Cooper A, Guest CB, Mazer CD, Mehta S, Stewart TE, Kudlow P, Cook D, Slutsky AS, Cheung AM, Canadian Critical Care Trials Group (2011) Functional disability 5 years after acute respiratory distress syndrome. New Engl J Med 364:1293-1304

15. Weled BJ, Adzhigirey LA, Hodgman TM, Brilli RJ, Spevetz A, Kline AM, Montgomery VL, Puri N, Tisherman SA, Vespa PM, Pronovost PJ, Rainey TG, Patterson AJ, Wheeler DS, Task Force on Models for Critical Care (2015) Critical care delivery: the importance of process of care and ICU structure to improved outcomes: an update from the American College of critical care medicine task force on models of critical care. Crit Care Med 43:1520-1525 